\title{
Neuropathology and Virus in Brain of SARS-CoV-2 Infected Non-Human Primates
}

Ibolya Rutkai

Tulane University

Meredith Mayer

Tulane University

Linh Hellmers

Tulane University

Bo Ning

Tulane University

Zhen Huang

Tulane University

Christopher Monjure

Tulane National Primate Research Center

Carol Coyne

Tulane University

Rachel Silvestri

Tulane University

Nadia Golden

Tulane University

Krystle Hensley

Tulane University

Kristin Chandler

Tulane University

Gabrielle Lehmicke

Tulane National Primate Research Center

\section{Gregory Bix}

Tulane University https://orcid.org/0000-0002-8969-9553

\section{Nicholas Maness}

Tulane National Primate Research Center

Kasi Russell-Lodrigue

Tulane National Primate Research Center https://orcid.org/0000-0003-3719-0779

Tony $\mathrm{Hu}$

Tulane University

Chad Roy 
Tulane University https://orcid.org/0000-0002-1710-6974

\section{Robert Blair}

Tulane National Primate Research Center https://orcid.org/0000-0001-9616-9414

\section{Rudolf Bohm}

Tulane National Primate Research Center

\section{Lara Doyle-Meyers}

Tulane National Primate Research Center

\section{Jay Rappaport}

Tulane National Primate Research Center

\section{Tracy Fischer ( $\boldsymbol{\nabla}$ tfischer1@tulane.edu )}

Tulane National Primate Research Center https://orcid.org/0000-0003-4859-3776

\section{Research Article}

Keywords: COVID-19, coronavirus, complications, neurological manifestations, neurological disease

Posted Date: August 24th, 2020

DOl: https://doi.org/10.21203/rs.3.rs-59871/v1

License: (c) (1) This work is licensed under a Creative Commons Attribution 4.0 International License. Read Full License

Version of Record: A version of this preprint was published at Nature Communications on April 1st, 2022. See the published version at https://doi.org/10.1038/s41467-022-29440-z. 


\section{Neuropathology and Virus in Brain of SARS-CoV-2 Infected Non-Human Primates}

2

3 Ibolya Rutkai1, Meredith G. Mayer2, Linh M. Hellmers2, Bo Ning, Zhen Huang, Christopher J. Monjure2, Carol 4 Coyne2, Rachel Silvestriz, Nadia Golden,, Krystle Hensley2, Kristin Chandler2, Gabrielle Lehmicke2, Gregory J. 5 Bix1, Nicholas J. Maness2, Kasi Russell-Lodrigue2, Tony Y. Hu3, Chad J. Roy2, Robert V. Blair2, Rudolf Bohm2, 6 Lara A. Doyle-Meyers2, Jay Rappaport2, and Tracy Fischer2, *

8 1Department of Neurosurgery, Clinical Neuroscience Research Center, Tulane University School of Medicine, 9 New Orleans, LA 70112, USA

10 2Tulane National Primate Research Center, 18703 Three Rivers Road, Covington, LA 70433, USA

11 3Center for Cellular and Molecular Diagnostics, Tulane University School of Medicine, 1430 Tulane Ave., New 12 Orleans, LA 70112, USA

15 *To whom correspondence should be addressed:

17 Tracy Fischer, $\mathrm{PhD}$

18 Associate Professor

19 Division of Comparative Pathology

20 Tulane National Primate Research Center

2118703 Three Rivers Road

22 Building 2, Room 111B

23 Covington, LA 70433

24 tfischer1@tulane.edu

25 Tel. 985-871-6394 


\section{ABSTRACT}

29 Neurological manifestations are a significant complication of coronavirus infection disease-19 (COVID-19).

30 Understanding how COVID-19 contributes to neurological disease is needed for appropriate treatment of infected

31 patients, as well as in initiating relevant follow-up care after recovery. Investigation of autopsied brain tissue has

32 been key to advancing our understanding of the neuropathogenesis of a large number of infectious and non33 infectious diseases affecting the central nervous system (CNS). Due to the highly infectious nature of the etiologic 34 agent of COVID-19, severe acute respiratory syndrome coronavirus 2 (SARS-CoV-2), there is a paucity of 35 tissues available for comprehensive investigation. Here, we show for the first time, microhemorrhages and neuropathology that is consistent with hypoxic injury in SARS-CoV-2 infected non-human primates (NHPs).

37 Importantly, this was seen among infected animals that did not develop severe respiratory disease. This finding 38 underscores the importance of vaccinating against SARS-CoV-2, even among populations that have a reduced 39 risk for developing of severe disease, to prevent long-term or permanent neurological sequelae. Sparse virus 40 was detected in brain endothelial cells but did not associate with the severity of CNS injury. We anticipate our 41 findings will advance our current understanding of the neuropathogenesis of SARS-CoV-2 infection and 42 demonstrate SARS-CoV-2 infected NHPs are a highly relevant animal model for investigating COVID-19 43 neuropathogenesis among human subjects. 
Multiple and continuing reports demonstrate a substantial number of patients with coronavirus disease 2019

(COVID-19) develop new onset neurological manifestations. Indeed, several case reports have identified

neurological complications as the initial presentation of severe acute respiratory syndrome coronavirus 2 (SARS-

CoV-2) infection, particularly among those who develop stroke 1 -3. Among the more urgent COVID-19-associated neurological presentations, stroke, meningoencephalitis, and hemorrhagic necrotizing encephalopathies have been associated with more severe disease $2,4-6$, however, even comparatively mild symptoms, such as dizziness or unresolving headache 4,7 , may be indicative of neuropathological processes in the context of infection and disease. While damage to the central nervous system (CNS) of patients with COVID-19 is increasingly evident, the neuropathogenesis remains unclear. Here, we provide the first comprehensive assessment of brain pathology associated with SARS-CoV-2 infection, using two non-human primate (NHP) models of infection and disease. This work reveals multiple microhemorrhages and pathology consistent with hypoxic injury with limited infection of brain vasculature in SARS-CoV-2 infected NHPs and provides key insights into SARS-CoV-2associated neuropathogenesis.

\section{Neuronal injury and apoptosis}

Eight adult NHPs, including four Rhesus macaques (RM), 13-15 years of age, and four wild-caught African green monkeys (AGMs), approximately 16 years of age, were inoculated with the 2019-nCoV/USA-WA1/2020 strain of SARS-CoV-2 \& via a multi-route mucosal or aerosol challenge (Extended Data Table 1). Two animals of each species were inoculated via aerosol and two by multi-route exposure. Multi-route mucosal exposure included conjunctival, nasal, pharyngeal, and intratracheal routes. All animals developed infection within the first week of exposure, as demonstrated by RT-qPCR of nasal swabs at 7 days post infection (Extended Data Fig. 5). Apart from RM3 and RM4, detectable virus was seen in nasal swabs of all animals up to and including the day of necropsy, approximately 4 weeks post exposure. No differences in infection were noted between the two inoculation strategies (Extended Data Fig. 5). All animals survived to study endpoint, with the exception of AGM4 and AGM2. At 8 days post infection, AGM4 was found recumbent and marginally responsive to stimuli.

This animal also presented with dyspnea/tachypnea (respiratory rate of 72 breaths per minute), hypothermia (< $\left.32.2^{\circ} \mathrm{C}\right)$, and hypoxemia [blood oxygen saturation $\left(\mathrm{SpO}_{2}\right)=77 \%$ ] and was euthanized. At 22 days post infection, 
shortly before its scheduled study endpoint, AGM2 developed severe tachypnea, hypothermia, and hypoxemia, with a respiratory rate of 96 breaths per minute and $\mathrm{SpO}_{2}=77 \%$ and was subsequently euthanized.

Seven regions of the central nervous system (CNS), including frontal, parietal, occipital, and temporal lobes, basal ganglia, cerebellum, and brainstem were collected at necropsy and investigated by histopathological and who had been euthanized due to non-SARS-CoV-2 related illness, were included as age-matched controls. Summary of the pathological findings in brain for all animals are included in Extended Data Table 2.

Hematoxylin and eosin (H\&E) staining revealed marked changes in neuronal morphology, which was most commonly observed in cerebellum and brainstem (Fig. 1a, b). Neuronal degeneration was characterized by pyknotic and karyorrhectic neurons with shrunken cytoplasm and vacuolation in the surrounding neuropil (Fig. 1a, b; Extended Data Fig. 1b, c). The cerebellum contained multifocal regions of degenerate Purkinje neurons that exhibited cellular blebs and debris and cytoplasmic vacuoles (Fig. 1a; Extended Data Fig. 1b). Contiguous with regions of degenerate Purkinje cells, neurons and glia within the molecular and granular layers appeared pyknotic with condensed basophilic nuclei (Fig. 1a). Similar morphologic changes were noted in glial cells adjacent to apoptotic neurons in the brainstem (Fig. 1b). In both brainstem and cerebellum, neurons are seen at various stages of nuclear dissolution (Fig. 1a, b; Extended Data Fig. 1b, c). Degeneration of Purkinje cells was confirmed with FluoroJade C (Extended Data Fig. 1d-f).

While vacuolation was frequently observed in the cerebellar gray and white matter (Fig. 1C; Extended Data Fig. 2a, b), significant demyelination was not a major finding in this study. Luxol Fast Blue (LFB) revealed areas of myelin pallor, suggestive of oligodendrocyte injury and/or loss, in the cerebellum of RM3 and occipital lobe of AGM1 (Extended Data Fig. 2c, d).

Given the prominent morphologic changes noted within Purkinje cells, we sought to identify the mechanisms underlying these degenerative changes by investigating tissues for the presence of cleaved caspase 3 , the activated form of this key executioner of apoptosis. Cleaved caspase 3 was seen in at least one CNS region from seven of the eight study animals and both archival controls (Fig. 1d, e; Extended Data Fig. 3). Three animals, RM1, AGM1, and AGM3 showed positivity in more than one region, while RM2 was positive in all regions examined (Fig. 1f; Extended Data Fig. 3). Although cleaved caspase 3 was observed in all CNS regions 
evaluated, it was most frequently elevated in basal ganglia, brainstem, and cerebellum (Extended Data Fig. 3).

In cerebellum, cytoplasmic and nuclear cleaved caspase 3 was predominantly restricted to cells within and proximal to the Purkinje cell layer (Fig. 1d; Extended Data Fig. 1c). Other CNS regions, including brainstem, had foci of cleaved caspase 3 positivity (Fig. 1e). In comparison to the study animals, both control animals had a markedly lower frequency of positive cells (Fig. 1f; Extended Data Fig. 3). Interestingly, cleaved caspase 3 was not detected in any CNS region examined from AGM4, who was euthanized at 8 days post infection due to advanced illness. This may suggest programmed cell death in the CNS occurs later in the disease process.

\section{Brain microhemorrhages}

Microhemorrhages, as suggested by the presence of erythrocyte extravasation into the brain parenchyma, were seen in study animals but were restricted to brainstem, cerebellum, and basal ganglia in the examined tissues (Fig. 2a-d; Extended Data Table 2). Microhemorrhages were observed with and without ischemic injury of adjacent tissues, characterized by localized/regional pallor (Fig. 2a-d). Two microhemorrhages were seen in the brainstem of each of the two control animals and a single one was identified in cerebellum of one control animal (Extended Data Fig. 4; Extended Data Table 2); however, these lacked the size and frequency of the microhemorrhages observed in the SARS-CoV-2 infected animals.

Accumulation of microhemorrhages in the CNS occurs with aging and are seen most frequently in deep brain structures, including brainstem, cerebellum, and basal ganglia 9. To investigate the potential for pre-existing microhemorrhages in our study animals, all brain regions were stained with Gomori Prussian Blue (G Fe), which can aid in the identification of old cerebral hemorrhages through ferric iron ( $\left.\mathrm{Fe}_{3+}\right)$ deposition. Three animals, RM3, RM2, and AGM2, demonstrated blood vessel-associated iron deposition with minimal parenchymal involvement within the basal ganglia (Fig. 3a, b). In comparison, more recent microhemorrhages were also seen in basal ganglia, as suggested by the absence of iron with Prussian Blue staining (Fig. 3c, d).

With the exception of RM3, all study animals had multiple acute microhemorrhages, characterized by extravasation of erythrocytes into the brain parenchyma in the absence of $\mathrm{Fe}_{3+}$ deposition. This was most frequently observed in brainstem and cerebellum, as compared to the other CNS regions investigated. Interestingly, RM3 had a lower frequency of acute microhemorrhages and caspase 3 positivity. As such, the 
neuropathology observed in this animal may be associated with age-related changes in the CNS that are commonly observed in humans and NHPs.

\section{Chronic hypoxemia}

Microhemorrhages and ischemia appear to play a central role in neuronal injury observed in this study. The brain is a highly metabolic organ with a limited capacity for energy storage. Due to the significant energy demands of the brain and neurons, a prolonged reduction in blood flow and concomitant reduction in oxygen and glucose can be detrimental to neuronal vitality, in addition to the resulting neurotoxicity of erythrocyte breakdown products and inflammation. Of particular interest is the finding that AGM4, who was found recumbent and minimally responsive to stimuli at 8 days post infection, had a substantial number of microbleeds in the cerebellum, basal ganglia, and brainstem (Extended Data Table 2). These findings suggest AGM4 suffered multiple acute microhemorrhages that may have contributed to her rapid decline. Alternatively, AGM4's rapid pulmonary decline may have promoted end stage microhemorrhages. The timing of acute microhemorrhages in the disease process is unclear and warrants further investigation.

In addition to localized ischemic injury, all study animals experienced variations in $\mathrm{SpO}_{2}$ that fluctuated between 89 and $99 \%$ but stayed below 95\% for the majority of animals over the study course (Fig. 2f). Correspondingly, blood carbon dioxide $\left(\mathrm{CO}_{2}\right)$ ranged from 24 to $33 \mathrm{mEq} / \mathrm{L}$, remaining above the physiological range for the majority of the study animals (Fig. 2e). While these levels are not immediately alarming, they may suggest mild hypoxemia and impaired gas exchange in the lungs. Notably, blood $\mathrm{CO}_{2}$ remained within the physiological range the longest for RM3, who exhibited milder neuropathology compared to other SARS-CoV-2 infected animals. The brain requires aerobic metabolism of glucose for ATP production and any prolonged or intermittent reductions of blood $\mathrm{O}_{2}$ may contribute to localized CNS hypoxia and energy failure. Even minor reductions in oxygen may promote injury, particularly among neurons, which appear to have suffered the greatest insult in this study. In support of this notion, large regions of Purkinje cells, which are especially vulnerable to hypoxic insult 10,11, as well as cells in their immediate proximity, appear degenerate or committed to undergoing apoptosis. 


\section{Rare virus in brain-associated endothelium}

Using an antibody against SARS-CoV-2 nucleocapsid (SARS-N), IHC studies revealed marginal virus infection of brain vasculature (Fig. 4a). Virus was detected within the basal ganglia, cerebellum, and/or brainstem of all animals and was seen less often within the temporal, parietal, and occipital lobes (Extended Data Table 2). Suspected endothelial cell infection was confirmed by colocalization immunofluorescence (IF) of SARS-N with von Willebrand Factor (vWF; Fig. 4b; Extended Data Fig. 5c - f). This is in agreement with a previous study of human biopsy tissues that demonstrated the principal receptor for SARS-CoV-2, angiotensin-converting enzyme 2 (ACE2), is expressed by endothelial cells throughout the body, including brain 12,13. Virus infection of cells within the brain parenchyma was not convincingly demonstrated. Brain tissues were also found to be negative for the presence of viral inclusion bodies by Machiavello staining.

Using a highly sensitive CRISPR-based fluorescent detection system (CRISPR-FDS) 14, virus was not identified in the cerebrospinal fluid (Fig. 4c), consistent with most findings among human subjects, except in rare cases of encephalitis 15-17. In contrast, this method detected limited viral RNA in whole brain, frozen at the time of necropsy, that was largely representative of our IHC/IF findings (Fig. 4d). Similar to our findings in fixed tissues, virus was more frequently observed in basal ganglia, cerebellum, and brain stem. CRISPR-FDS analysis also revealed viral RNA in the frontal lobe of one animal, AGM4, which was not convincingly seen by IHC/IF for this region in any study animal. This may reflect differences in sampling error that is inherently present in the two methods, where the amount of tissue used for the CRISPR-FDS studies is greater than that used in IHC, and/or extracerebral virus that may have been present in the blood vessel lumen. Together, these findings demonstrate limited SARS-CoV-2 infection in brain-associated endothelial cells in deep brain structures of NHPs, even in the absence of severe disease or overt neurological symptoms.

\section{Discussion}

The mechanisms underlying SARS-CoV-2-associated neurological complications is unclear and most likely, complex. Hypoxia, chronic hypoxemia, exaggerated and prolonged immune response, and direct virus injury to endothelial cells may all play a contributing role. In addition, neuropathogenesis has been difficult to investigate, due to the scarcity of brain tissues acquired at autopsy from human subjects who succumbed to disease. Here, we show for the first time, substantial pathological changes in brain of SARS-CoV-2 infected NHPs that are 
compatible with hypoxic-ischemic injury, involving deep brain structures, including brainstem, cerebellum, and basal ganglia. Large regions of injured neurons at various stages of nuclear dissolution were observed throughout the CNS compartment. This does not appear to be a direct consequence of virus infection, as only limited virus was seen in brain vasculature but did not appear to involve parenchymal cells. Instead, neuronal injury and death most likely occur as a result of energy failure, which is an early consequence of hypoxic-ischemic events. Multiple microhemorrhages, microinfarcts, and hypoxemia appear to play a role in neuronal injury and death observed in these animals.

Our findings in NHPs are largely in agreement with the limited autopsy studies of brain from human subjects 18,19. It is reasonable to anticipate that similar processes occur among human subjects, which would account for many of the neurological manifestations observed among infected individuals, including seizures, ataxia, and altered mental status. Neuropathological complications may also contribute to worsening disease. For example, damage to the brainstem, which modulates the respiratory cycle by regulating inspiratory and expiratory muscle activity, may contribute to worsening respiratory distress and failure in patients with COVID-19. Additional studies, employing relevant animal models, are warranted and likely to reveal important insight into human disease.

While SARS-CoV-2 neuropathogenic processes are poorly understood, this work, employing a highly relevant animal model, provides key insights that may inform further studies, as well as treatment modalities. Importantly, varying degrees of neuropathology was seen in infected NHPs, even in the absence of severe respiratory disease. This may suggest hypoxic brain injury is a common complication of SARS-CoV-2 infection and COVID-19. Neuronal degeneration and activation of caspase 3 observed in this study supports this notion and indicates non-reversible neuronal injury. Our findings and conclusions presented herein suggest the need for long-term neurological follow-up of persistently symptomatic convalescent patients, especially those who suffered severe disease. 


\section{Materials and Methods}

\section{Ethics and biosafety statement}

All animal studies were approved by the Tulane University Institutional Animal Care and Use Committee (IACUC) and carried out in the Regional Biocontainment Laboratory at the Tulane National Primate Research Center (TNPRC) within an animal biosafety level 3 (ABSL3) facility. The TNPRC is fully accredited by the AAALAC. All animals were cared for in accordance with the Institute for Laboratory Animal Research (ILAR) Guide for the Care and Use of Laboratory Animals, 8th edition. The Tulane University Institutional Biosafety Committee (IBC) approved all procedures for sample handling, inactivation, and removal from BSL3 containment.

\section{Animal study design}

A total of eight non-human primates, including four Indian-origin Rhesus macaques (RMs; ages 13-16 years) and African green monkeys (AGMs) of Caribbean origin (all approximately 16 years of age), were inoculated with SARS-CoV-2 strain 2019-nCoV/USA-WA1/2020 (MN985325.1) (Extended Data Table 1). This strain was isolated from the first confirmed SARS-CoV-2 case in the United States and deposited by the Centers for Disease Control 8.

All RMs were acquired from the Tulane National Primate Research Center specific pathogen-free breeding colony and confirmed negative for simian type D retrovirus (SRV), simian immunodeficiency virus (SIV), simian T cell lymphotropic virus type 1 (STLV1), measles virus (MV), Macacine herpesvirus 1 (MHV1/B virus), and tuberculosis (TB). The AGMs were wild-caught and also confirmed negative for SRV, SIV, STLV, MV, and TB. The AGMs were housed at the Center for over a year before assignment to this study. All animals were tested and found negative for SARS-CoV-2 (antibody and virus) prior to experimental infection.

Two routes of virus exposure, multi-route mucosal and aerosol, were employed to mimic major routes of infection among humans. Two animals from each species were randomly subjected to the different routes of exposure for a total of four animals in each species challenge group. Multi-route exposure included conjunctival, nasal, pharyngeal, and intratracheal routes for a cumulative dose of $3.61 \times 10_{6}$ PFU (plaque-forming unit). Animals exposed to virus by aerosol received an approximate inhaled dose of $2 \times 10_{3}$ TCID 50 ( $50 \%$ tissue culture infectious dose). Study animals were euthanized for necropsy at 24-28 days post infection, unless humane endpoints 
required euthanasia at an earlier time (Extended Data Table 1). Postmortem examination was performed by a board-certified veterinary pathologist (RVB).

\section{Quantification of Nasal Swab SARS-CoV-2 RNA}

Nasal swab specimens were collected in $200 \mu \mathrm{L}$ DNA/RNA Shield (Zymo Research) and extracted for viral RNA (vRNA) using the Quick-RNA Viral kit (Zymo Research). Viral RNA Buffer (Zymo) was dispensed directly to the swab in the DNA/RNA Shield (Zymo). A modification to the manufacturers' protocol was to insert the swab directly into the spin column to centrifugate, allowing all the solution to cross the spin column membrane. The vRNA was eluted $(45 \mu \mathrm{L})$, from which $5 \mu \mathrm{L}$ was added to a $0.1 \mathrm{~mL}$ fast 96 -well optical microtiter plate format (Thermo Fisher) for a $20 \mu \mathrm{L}$ RT-qPCR reaction. The RT-qPCR reaction used TaqPath 1-Step Multiplex Master Mix (Thermo Fisher) along with the 2019-nCoV RUO Kit (IDTDNA), a premix of forward and reverse primers, and a FAM labeled probe targeting the $\mathrm{N} 1$ amplicon of $\mathrm{N}$ gene of SARS2-nCoV19 (accession MN908947). The reaction master mix was added using an X-Stream repeating pipette (Eppendorf) to the microtiter plates. Loaded plates were covered with optical film (Thermo Fisher), vortexed, and pulse centrifuged. The RT-qPCR reaction employed the following program: UNG incubation at $25^{\circ} \mathrm{C}$ for 2 minutes, $\mathrm{RT}$ incubation at $50^{\circ} \mathrm{C}$ for 15 minutes, and an enzyme activation at $95^{\circ} \mathrm{C}$ for 2 minutes, followed by 40 cycles of denaturation at $95^{\circ} \mathrm{C}$ for 3 seconds and annealing at $60^{\circ} \mathrm{C}$ for 30 seconds. Fluorescence signals were detected with an Applied Biosystems QuantStudio 6 Sequence Detector. Data were captured and analyzed with Sequence Detector Software v1.3 (Applied Biosystems). Viral copy numbers were calculated by plotting Cq values obtained from unknown (i.e., test) samples against a standard curve representing known viral copy numbers. The limit of detection of the assay was 10 copies per reaction volume. A 2019-nCoV positive control (IDTDNA) were analyzed in parallel with every set of test samples to verify that the RT-qPCR master mix and reagents were prepared correctly to produce amplification of the target nucleic acid. A non-template control (NTC) was included in the qPCR to ensure that there was no cross-contamination between reactions.

\section{Immunohistochemistry}

Immunohistochemistry was performed on $5 \mu \mathrm{m}$ zinc formalin-fixed paraffin-embedded (FFPE) brain sections, as described 20. Sections were deparaffinized in xylenes and rehydrated through an ethanol series ending in distilled water. Heat-mediated antigen retrieval was carried out in a vacuum oven with Tris-EDTA buffer (10mM 
254

255

256

257

258

259

260

261

262

263

264

265

266

267

268

269

270

271

272

273

274

275

276

277

278

279

Trizma base, $1 \mathrm{mM}$ EDTA, $0.05 \%$ Tween 20 , pH 9.0) or sodium citrate buffer (10mM sodium citrate, $0.05 \%$ Tween 20, $\mathrm{pH}$ 6.0). All washes were performed using tris buffered saline containing Tween 20 (TTBS; 0.1M Trizma base, $0.15 \mathrm{M} \mathrm{NaCl}, 0.1 \%$ Tween 20, $\mathrm{pH}$ 7.4). Following antigen retrieval, tissues were blocked with $20 \%$ normal horse or goat serum. Endogenous biotin was blocked with Avidin-Biotin Solution (Vector Labs). Titrated primary antibodies included anti-cleaved caspase 3 (rabbit polyclonal, 1:250, Abcam, ab2302), anti-von Willebrand Factor (rabbit EPR12010, 1:62.5, Abcam, ab179451), and anti-SARS-CoV-2 nucleocapsid (rabbit polyclonal, 1:125, Novus, NB100-56576). Tissues were incubated with primary antibody overnight at room temperature and detected using the appropriate biotinylated secondary antibody and alkaline phosphatase-Vector Red according to manufacturer instructions (Vector Labs). Tissues were counterstained with Mayer's hematoxylin and coverslipped.

Double-labeling of $5 \mu \mathrm{m}$ FFPE brain tissue was performed by sequential application of primary antibodies with their corresponding secondary, as described 21. SARS-CoV-2 nucleocapsid was detected with Alexa Fluor 555 (1:500, Invitrogen). Von Willebrand Factor was detected with Alexa Fluor 488 (1:500, Invitrogen). Controls consisted of brain tissue incubated in blocking buffer only, tissue incubated with one primary and the corresponding secondary antibody, and tissue incubated with fluorophore-conjugated secondaries only. Tissues were coverslipped with Vectashield $囚$ HardSet ${ }^{\mathrm{TM}}$ Antifade mount with DAPI (Vector Labs).

\section{Hematoxylin and eosin}

Deparaffinized and rehydrated slides were taken through Hemalast and hematoxylin, followed by differentiator and bluing solutions. After which, slides were dehydrated in 95\% EtOH and stained with eosin. Stained slides were dehydrated, cleared, and coverslipped.

\section{Luxol Fast Blue}

Slides were deparaffinized and rehydrated through $95 \% \mathrm{EtOH}$, then incubated in warmed $0.1 \%$ Luxol Fast Blue solution. Afterward, slides were washed, dipped in $0.05 \%$ lithium carbonate, differentiated in $70 \% \mathrm{EtOH}$, and rinsed. Following a check under microscope, the slides were oxidized in $0.5 \%$ periodic acid solution, then immersed in Schiff's reagent before rinsing, dehydration, clearing and coverslipping. 


\section{Gomori Prussian Blue}

$5 \mu \mathrm{m}$ FFPE brain sections were immersed in hydrochloric acid-potassium ferrocyanide solution and counterstained with nuclear fast red.

\section{Macchiavello's stain}

$5 \mu \mathrm{m}$ FFPE brain sections were immersed in $0.25 \%$ basic fuchsin, differentiated in $0.5 \%$ citric acid, washed, and counterstained in $1 \%$ methylene blue.

\section{FluoroJade C}

$5 \mu \mathrm{m}$ FFPE tissues were immersed in $0.06 \% \mathrm{KMNO}_{4}$ for 10 minutes and washed. Tissues were then immersed in $0.0002 \%$ Fluoro-Jade $\mathrm{C}$ (Histo-Chem) containing $0.1 \%$ acetic acid in the dark for 20 minutes, counterstained with 4',6-diamidino-2-phenylindole (DAPI), washed, and dried at $60^{\circ} \mathrm{C}$. Cleared tissues were coverslipped with DPX mount (Sigma).

\section{Imaging and quantitation}

Slides were scanned with the Axio Scan.Z1 digital slide scanner (Zeiss). Brightfield and fluorescent images were acquired using HALO (Indica Labs, v2.3.2089.70). Threshold and multiplex analyses were performed with HALO for non-biased quantitation of raw caspase 3 images, without processing. Colocalization of SARS-N and vWF was created in Photoshop (Adobe, v21.2.0) by overlaying the same image acquired through the appropriate fluorophore filter. Presented images were subjected to brightness or brightness and contrast enhancement, applied to the entire image.

\section{RNA isolation from whole tissues}

Dissected frontal lobe, basal ganglia, cerebellum, and brain stem were collected fresh and immediately frozen at necropsy. $1 \mathrm{~mL}$ of Trizol LS (Thermo Fisher) was added to $100 \mathrm{mg}$ of thawed tissue and homogenized in gentleMACS M tubes using a gentleMAC Dissociator (Miltenyi Biotec). The resulting lysate was then centrifuged at $3,000 \times \mathrm{g}$ for 5 minutes and supernatant transferred into a $2 \mathrm{~mL}$ microcentrifuge tube. An equal volume of ethanol (95-100\%) was added to the sample in Trizol LS (1:1) and mixed well. The resulting mixture was transferred to a Zymo-Spin III CG Column in a $2 \mathrm{~mL}$ collection tube (Zymo) and centrifuged for 30 seconds. The column was washed with RNA Wash Buffer (Zymo), followed by treatment with DNase I for 30 min to remove 
residual genomic DNA (gDNA). The column was washed with RNA Wash Buffer (Zymo) and RNA eluted with $45 \mu \mathrm{L}$ of DNase/RNase-free water (Thermo Fisher).

\section{CRISPR-based fluorescent detection system (CRISPR-FDS)}

CRISPR-FDS reaction was carried out as described previously 14. Isolated RNA samples were mixed with one-step RT-PCR mix containing 2X Platinum тм SuperFiтм RT-PCR Master Mix (Thermo Fisher), forward primer $(10 \mu \mathrm{M})$, reverse primer $(10 \mu \mathrm{M})$, SuperScriptтм IV RT Mix (Thermo Fisher), and nuclease-free water. Samples were then incubated in a T100 thermocycler (Bio-Rad) using a cDNA synthesis protocol, immediately followed by a DNA amplification protocol. CRISPR-FDS reactions were performed as follows: a sample RT-PCR reaction was transferred to a 96-well half-area plate and mixed with CRISPR reaction mixture containing 10X NEBuffer ${ }^{\mathrm{TM}}$ 2.1, gRNA (300nM), EnGen® Lba Cas12a $(1 \mu \mathrm{M})$, fluorescent probe $(10 \mu \mathrm{M})$, and nuclease-free water. After incubation at $37^{\circ} \mathrm{C}$ for 20 minutes in the dark, fluorescence signal was detected using SpectraMax i3x MultiMode Microplate Reader (Molecular Devices). A positive sample was defined as any specimen with a CRISPRFDS signal that was greater than the cut-off threshold of $3.6 \times 106 \mathrm{PL}$ intensity (a.u.).

\section{Statistics}

One-way analysis of variance (ANOVA) was performed with GraphPad Prism software, v8.4.3. Multiple comparison analyses with Tukey post-test identified differences between brain region, where $p \leq 0.05$ is considered significant. Data are expressed as mean \pm SD.

\section{Reporting summary}

A Nature Research Reporting Summary is linked to this paper.

\section{Data availability}

HALO annotations for quantification, scanned slides, and clinical data are available upon reasonable request.

\section{Acknowledgements}

This study was supported by P51OD011104, Pilot Grant from P51OD011104 to JR, Tulane startup funds and Emergent Ventures at the Mercatus Center, George Mason University Fast Grants for COVID-19 to TF, and Weatherhead Presidential Endowment to TYH. GJB was supported by Tulane startup funds. 


\section{Author Contributions}

$334 \mathrm{JR}, \mathrm{RB}, \mathrm{TF}, \mathrm{RVB}, \mathrm{LADM}, \mathrm{KRL}$, and CJR designed the study. RB and LADM provided animal clinical data 335 and interpretation. CJM, CC, RS, KH, KC, GL, and NJM participated in tissue acquisition and processing and 336 performed experiments. CRISPR-FDS data acquisition and interpretation were done by BN, ZH, and TYH. IR, 337 MGM, LMH, and TF performed experiments and acquired, analyzed and interpreted data. Necropsies and 338 sample collection were performed by RVB. Manuscript and figures were prepared by TF, IR, LMH, and MGM 339 and edited by JR, RB, GJB, RVB, and LADM. All authors have read and approved the manuscript.

341 Competing interests. The authors declare no competing interests. 


\section{References}

1 Oxley, T. J. et al. Large-Vessel Stroke as a Presenting Feature of Covid-19 in the Young. N Engl J Med, doi:10.1056/NEJMc2009787 (2020).

2 Mao, L. et al. Neurologic Manifestations of Hospitalized Patients With Coronavirus Disease 2019 in Wuhan, China. JAMA neurology, doi:10.1001/jamaneurol.2020.1127 (2020).

3 Avula, A. et al. COVID-19 presenting as stroke. Brain Behav Immun 87, 115-119, doi:10.1016/j.bbi.2020.04.077 (2020).

4 Romero-Sanchez, C. M. et al. Neurologic manifestations in hospitalized patients with COVID-19: The ALBACOVID registry. Neurology, doi:10.1212/WNL.0000000000009937 (2020).

5 Moriguchi, T. et al. A first case of meningitis/encephalitis associated with SARS-Coronavirus-2. Int J Infect Dis 94, 55-58, doi:10.1016/j.ijid.2020.03.062 (2020).

6 Poyiadji, N. et al. COVID-19-associated Acute Hemorrhagic Necrotizing Encephalopathy: Imaging Features. Radiology 296, E119-E120, doi:10.1148/radiol.2020201187 (2020).

7 Chen, T. et al. Clinical characteristics of 113 deceased patients with coronavirus disease 2019: retrospective study. BMJ 368, m1091, doi:10.1136/bmj.m1091 (2020).

8 Harcourt, J. et al. Severe Acute Respiratory Syndrome Coronavirus 2 from Patient with Coronavirus Disease, United States. Emerg Infect Dis 26, 1266-1273, doi:10.3201/eid2606.200516 (2020).

9 Roob, G. et al. MRI evidence of past cerebral microbleeds in a healthy elderly population. Neurology $\mathbf{5 2}$, 991-994, doi:10.1212/wnl.52.5.991 (1999).

10 Barenberg, P., Strahlendorf, H. \& Strahlendorf, J. Hypoxia induces an excitotoxic-type of dark cell degeneration in cerebellar Purkinje neurons. Neuroscience research 40, 245-254, doi:10.1016/s01680102(01)00234-6 (2001).

11 Bartschat, S., Fieguth, A., Konemann, J., Schmidt, A. \& Bode-Janisch, S. Indicators for acute hypoxia-an immunohistochemical investigation in cerebellar Purkinje-cells. Forensic Sci Int 223, 165-170, doi:10.1016/j.forsciint.2012.08.023 (2012).

12 Hamming, I. et al. Tissue distribution of ACE2 protein, the functional receptor for SARS coronavirus. A first step in understanding SARS pathogenesis. J Pathol 203, 631-637, doi:10.1002/path.1570 (2004). 
13 To, K. F. \& Lo, A. W. Exploring the pathogenesis of severe acute respiratory syndrome (SARS): the tissue distribution of the coronavirus (SARS-CoV) and its putative receptor, angiotensin-converting enzyme 2 (ACE2). J Pathol 203, 740-743, doi:10.1002/path.1597 (2004).

14 Huang, Z. et al. Ultra-sensitive and high-throughput CRISPR-p owered COVID-19 diagnosis. Biosens Bioelectron 164, 112316, doi:10.1016/j.bios.2020.112316 (2020).

15 Al Saiegh, F. et al. Status of SARS-CoV-2 in cerebrospinal fluid of patients with COVID-19 and stroke. J Neurol Neurosurg Psychiatry 91, 846-848, doi:10.1136/jnnp-2020-323522 (2020).

16 Benameur, K. et al. Encephalopathy and Encephalitis Associated with Cerebrospinal Fluid Cytokine Alterations and Coronavirus Disease, Atlanta, Georgia, USA, 2020. Emerg Infect Dis 26, doi:10.3201/eid2609.202122 (2020).

17 Huang, Y. H., Jiang, D. \& Huang, J. T. SARS-CoV-2 Detected in Cerebrospinal Fluid by PCR in a Case of COVID-19 Encephalitis. Brain Behav Immun 87, 149, doi:10.1016/j.bbi.2020.05.012 (2020).

18 Solomon, I. H. et al. Neuropathological Features of Covid-19. N Engl J Med, doi:10.1056/NEJMc2019373 (2020).

19 Kantonen, J. et al. Neuropathologic features of four autopsied COVID-19 patients. Brain Pathol, doi:10.1111/bpa.12889 (2020).

20 Tavazzi, E., Morrison, D., Sullivan, P., Morgello, S. \& Fischer, T. Brain inflammation is a common feature of HIV-infected patients without HIV encephalitis or productive brain infection. Curr HIV Res 12, 97-110 (2014).

21 Fischer-Smith, T. et al. CNS invasion by CD14+/CD16+ peripheral blood-derived monocytes in HIV dementia: perivascular accumulation and reservoir of HIV infection. J Neurovirol 7, 528-541 (2001). 

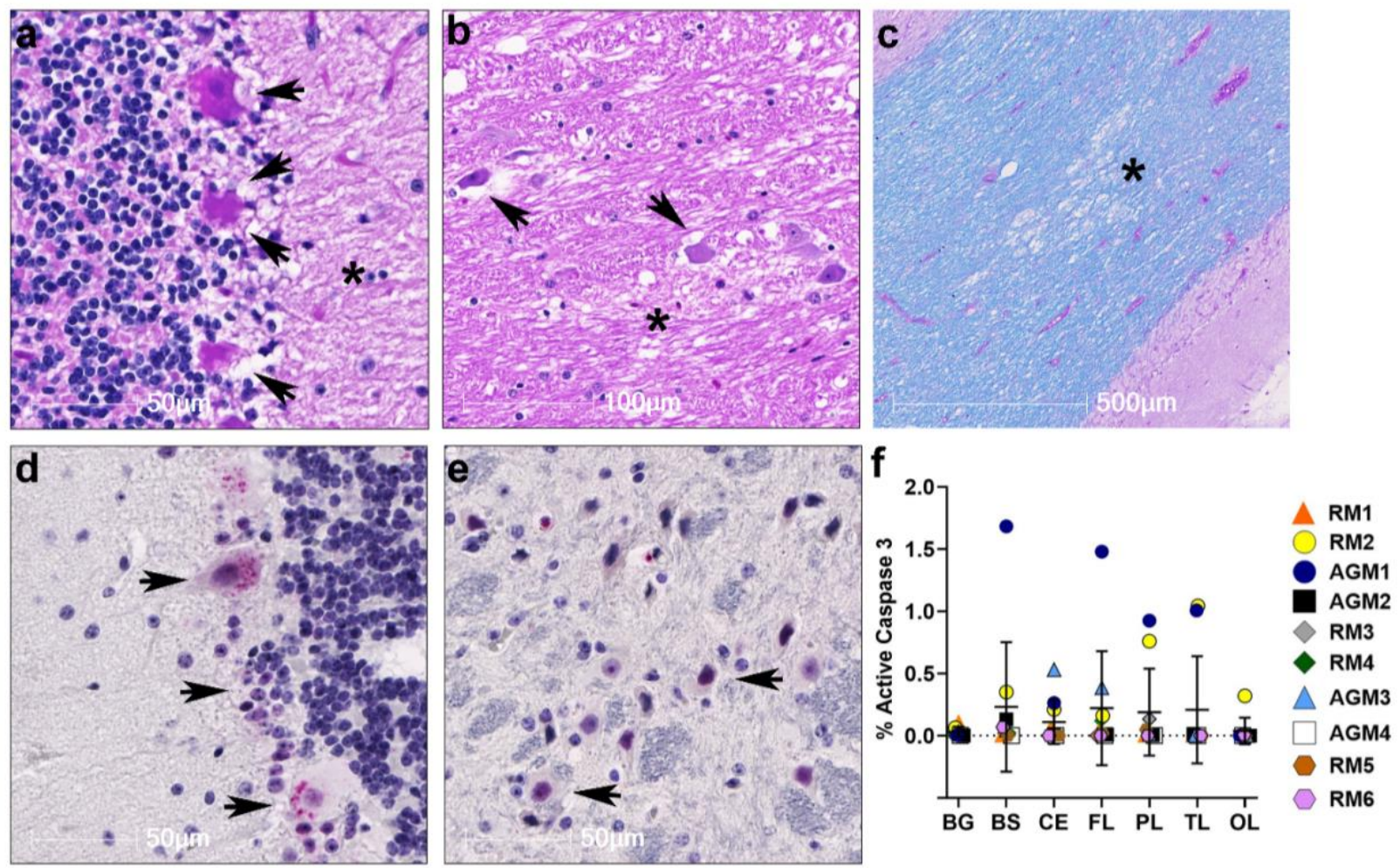

Figure 1. CNS cell death in SARS-CoV-2 infection. H\&E revealed death-associated neurons in cerebellum (a)

(a) and brainstem

(b). Arrows specify pyknotic and karyolitic cells (a and

b) and Purkinje cells with cellular blebs (a). Asterisks denote areas of tissue necrosis/vacuolation (a and b) and myelin vacuolation on Luxol Fast Blue stained cerebellum (c). Cleaved caspase 3 positive cells were found in cerebellum (d) and brainstem (e). Summary of cleaved caspase 3 frequency is organized by brain region (f). Data are expressed as mean \pm SD. BG - basal ganglia, BS brainstem, CE - cerebellum, FL - frontal lobe, PL - parietal lobe, TL - temporal lobe, OL - occipital lobe, RM - Rhesus macaque, AGM - African green monkey. 

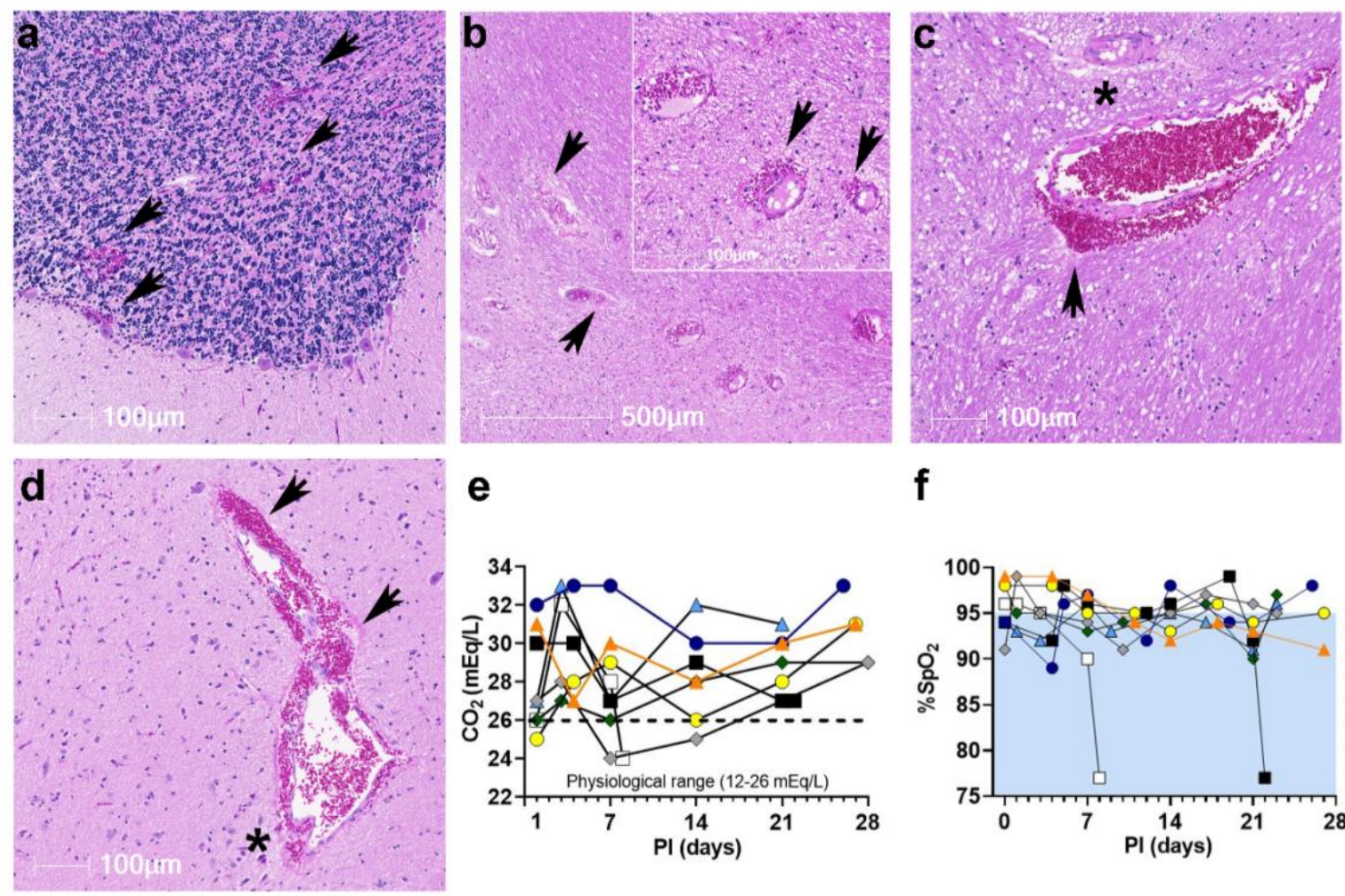

f

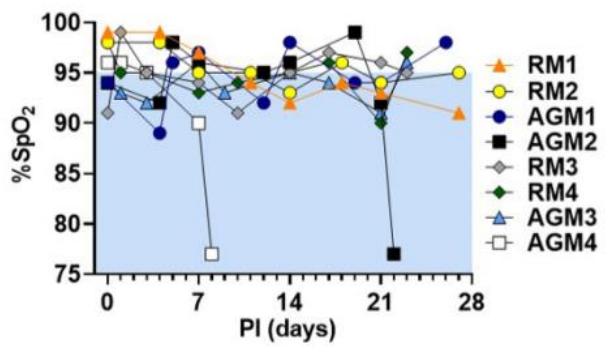

12 Figure 2. Multiple microhemorrhages in CNS of SARS-CoV-2 infected NHPs. H\&E of

cerebellum (a), brainstem (b and $\mathbf{c}$ ), and basal ganglia (d) revealed microhemorrhages, indicated by extravasation of erythrocytes into the brain parenchyma (arrows). Asterisks denote tissue injury around damaged blood vessels. Elevated blood $\mathrm{CO}_{2}(>26 \mathrm{mEq} / \mathrm{L})$ was detected throughout the study period for most animals. Dotted line indicates the upper limit of the physiological range for blood $\mathrm{CO}_{2}$ levels (e). $\mathrm{SpO}_{2}$ remained within the low normal range or under $95 \%$ for the majority of animals throughout the study. Blue shading indicates values $\leq 95 \%$ (f). RM - Rhesus macaque, AGM - African green monkey. 

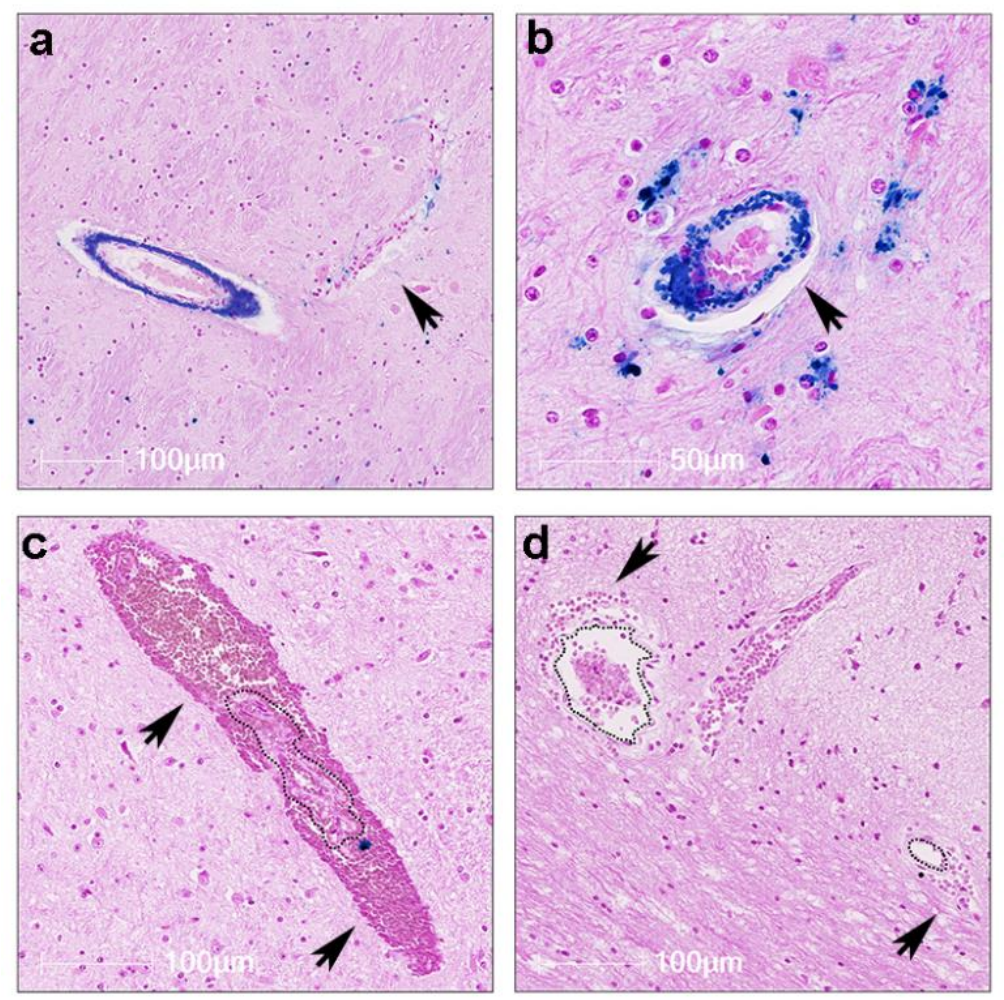

22 Figure 3. Pre-existing and acute vascular injury and microbleeds in brain. Gomori Prussian

23 Blue Fe (G Fe) revealed iron deposition within the basal ganglia (a and $\mathbf{b}$ ). Arrow denotes a normal

24 appearing blood vessel in close proximity to an iron positive vessel (a). Iron deposition was

25 predominantly seen in blood vessels with rare parenchymal involvement (b). Acute

26 microhemorrhages were also identified on G Fe stained basal ganglia, indicated by the absence

27 of G Fe positivity (arrows, $\mathbf{c}$ and $\mathbf{d}$ ). Dotted lines outline the blood vessel within the hemorrhagic

28 lesion (c and $\mathbf{d})$. 

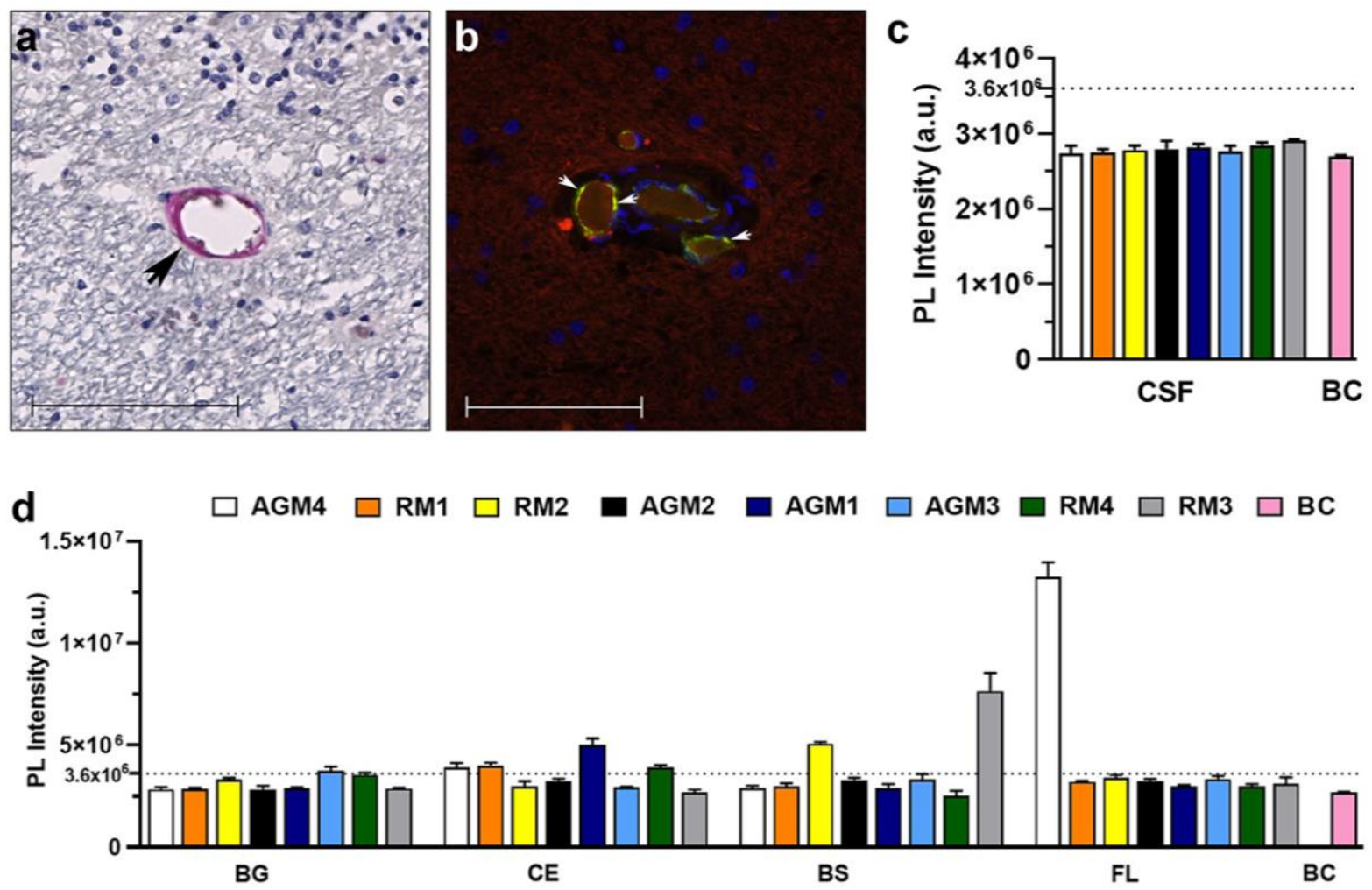

Figure 4. SARS-CoV-2 in the brain. IHC shows SARS-CoV-2 nucleocapsid (SARS-N) in

32 cerebellar blood vessels (a). Endothelial cell infection was confirmed by colocalization of SARS-

$33 \mathrm{~N}$ (red) and von Willebrand Factor (vWF) (green) (arrows, b). Scale bars $=100 \mu \mathrm{m}$. SARS-CoV-

342 RNA was undetectable in CSF (c) but was detected in different brain regions investigated (d).

35 Values above 3.6x106 PL intensity (dotted line) were considered positive. a.u. - arbitrary unit;

36 CSF - cerebrospinal fluid, BC - blank control, BG - basal ganglia, CE - cerebellum, BS -

37 brainstem, FL - frontal lobe, RM - Rhesus macaque, AGM - African green monkey. 

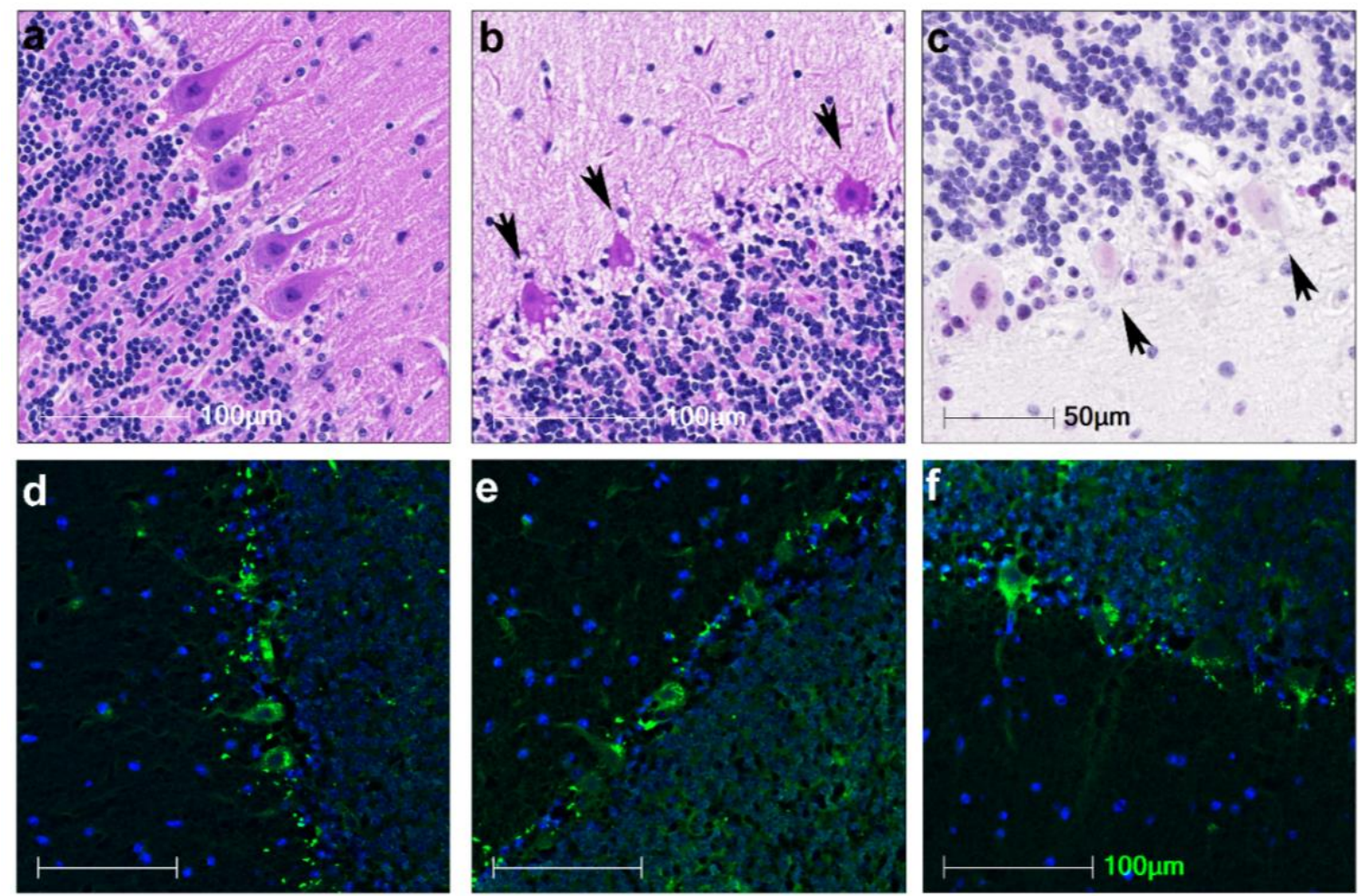

39 Extended Data Figure 1. Neuronal degeneration associated with SARS-CoV-2 infection.

40 Normal Purkinje cell and granular and molecular layer cell morphology of a non-SARS-CoV-2

41 infected animal (a). Representative cerebellar sections showed intracellular vacuolation and

42 nuclear fading and fragmentation (arrows, $\mathbf{b}$ and $\mathbf{c}$ ). Tissues shown were stained with H\&E (b) or

43 immunolabeled with caspase 3 (c). FluoroJade $\mathrm{C}$ revealed neuronal degeneration (bright green)

44 in cerebellum (d-f). Scale bars for $\mathbf{d}$ and $\mathbf{e}=100 \mu \mathrm{m}$. 

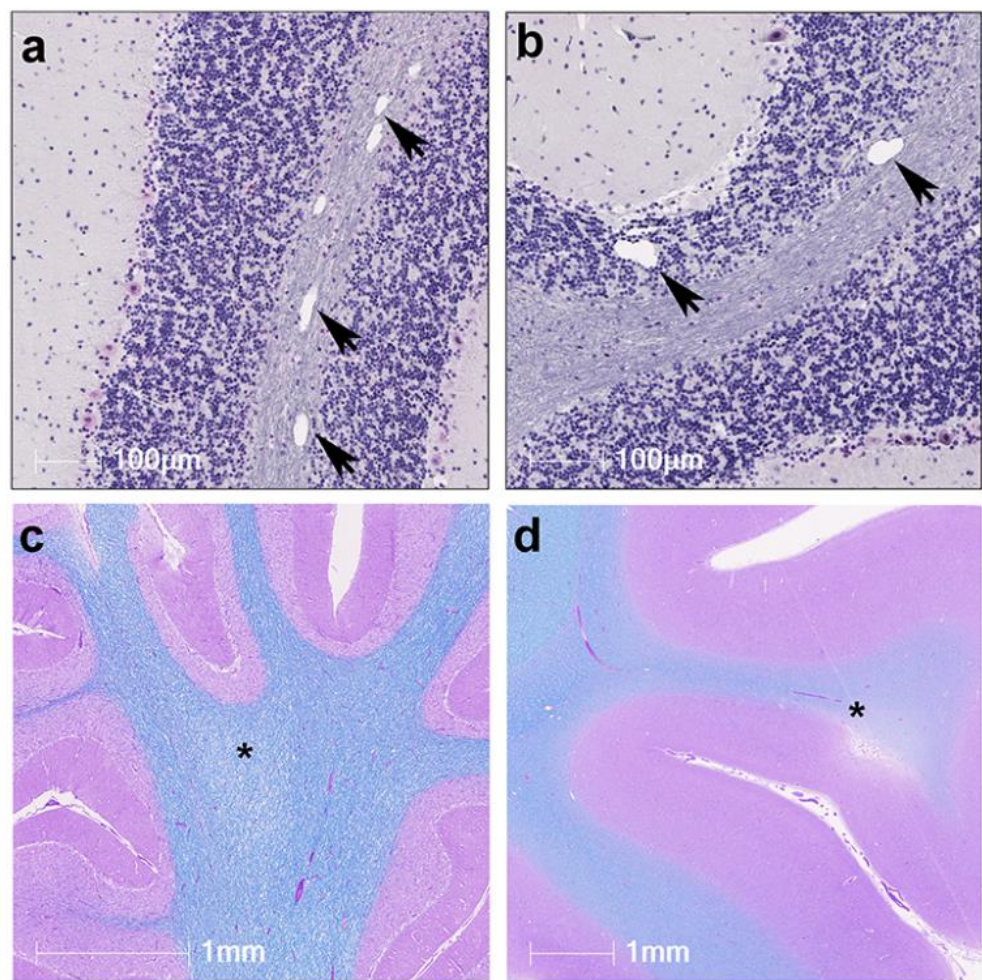

46 Extended Data Figure 2. Structural changes in brain of SARS-CoV-2 infected NHPs.

47 Representative images show vacuolation within white matter (a) and the granular layer (b) of

48 cerebellum, immunoassayed for caspase 3. Luxol Fast Blue revealed myelin loss in cerebellum

49 (c) and occipital lobe (d) (asterisks).

50

51 

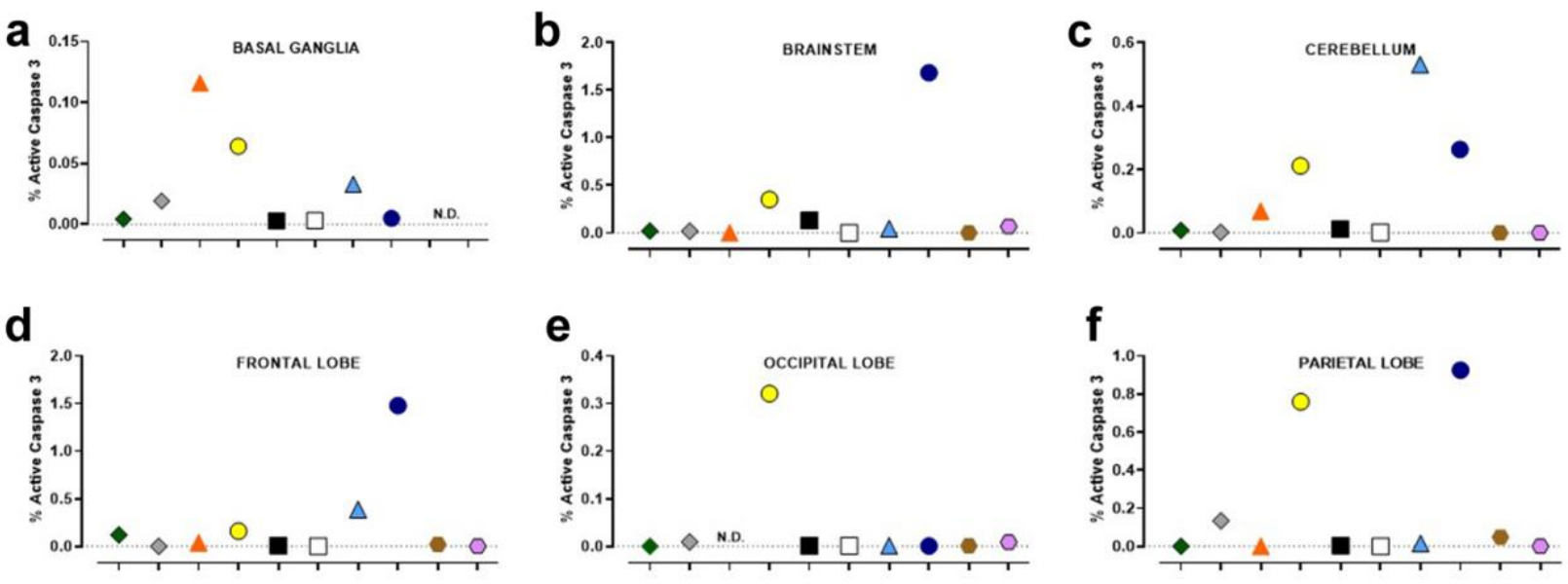

52

g

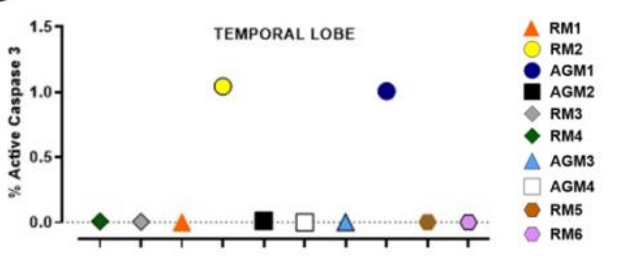

53 Extended Data Figure 3. Cleaved caspase 3 distribution in brain of SARS-CoV-2 infected

54 NHPs. Graphs show the regional distribution of quantitated caspase 3 positivity that is presented

55 in summation in Fig. 1f. RM - Rhesus macaque, AGM - African green monkey. 
57
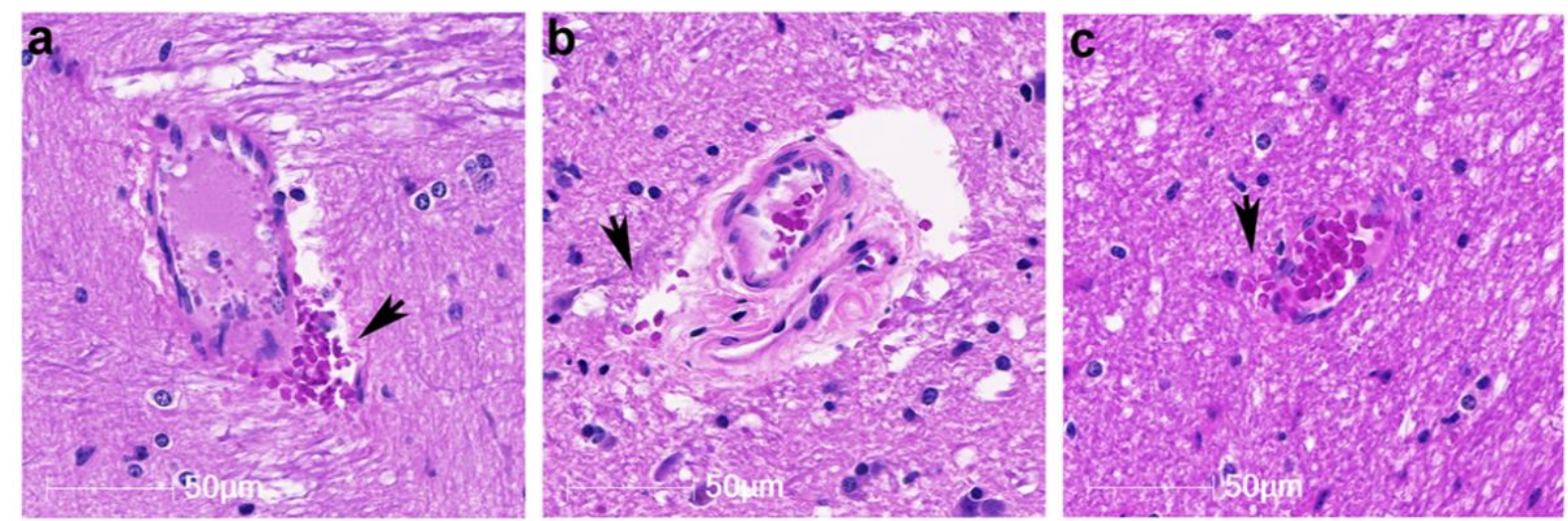

58 Extended Data Figure 4. Brain pathology in non-infected NHPs. H\&E stained brainstem and

59 cerebellum from non-infected animals revealed a limited number of microbleeds (a-c). Presented

60 images are representative of the less severe pathology observed, as compared to SARS-CoV-2

61 infected animals, which likely accumulate with normal aging.

62 


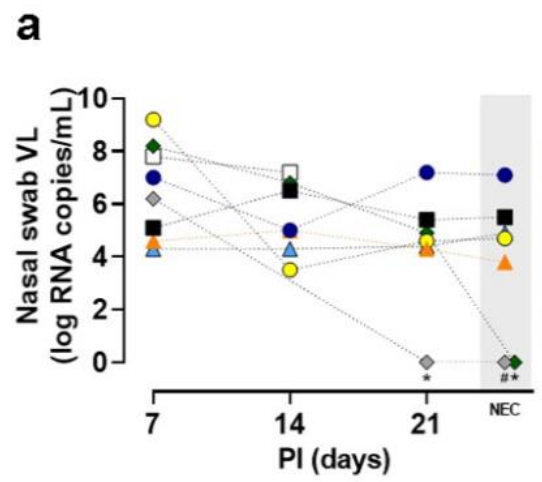

\begin{tabular}{|c|c|c|c|c|c|c|}
\hline \multirow{9}{*}{$\begin{array}{l}\text { RM1 } \\
\text { RM2 } \\
\text { RM3 } \\
\text { RM4 } \\
\text { AGM1 } \\
\text { AGM2 } \\
\text { AGM3 } \\
\text { AGM4 }\end{array}$} & & PI 7 & PI 14 & PI 21 & $\begin{array}{c}\text { VL at } \\
\text { necropsy }\end{array}$ & $\begin{array}{l}\text { Necropsy } \\
\text { (days PI) }\end{array}$ \\
\hline & RM1 & 4.6 & 5.0 & 4.3 & 3.8 & 27 \\
\hline & RM2 & 9.2 & 3.5 & 4.6 & 4.7 & 27 \\
\hline & AGM1 & 7.0 & 5.0 & 7.2 & 7.1 & 26 \\
\hline & AGM2 & 5.1 & 6.5 & 5.4 & 5.5 & 22 \\
\hline & RM3 & 6.2 & - & $\begin{array}{c}\text { not } \\
\text { detected }\end{array}$ & inconclusive & 28 \\
\hline & RM4 & 8.2 & 6.8 & 4.9 & not detected & 28 \\
\hline & AGM3 & 4.3 & 4.3 & 4.4 & 4.9 & 24 \\
\hline & AGM4 & 7.8 & - & - & 7.2 & 8 \\
\hline
\end{tabular}
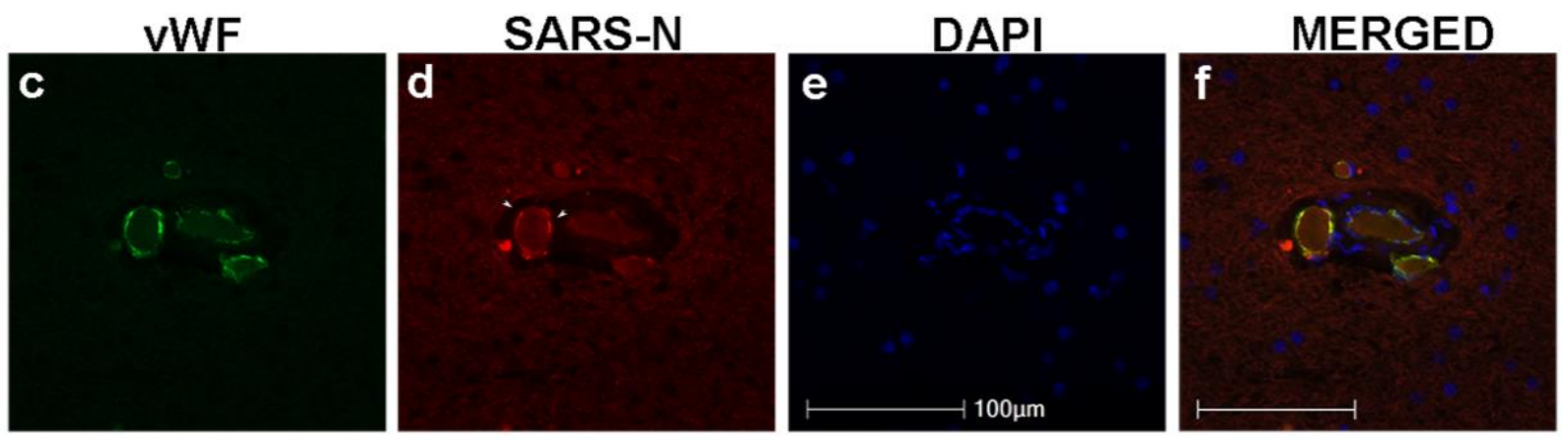

Extended Data Figure 5. SARS-CoV-2 infection of NHPs and virus detection in brain. SARS-

65 CoV-2 RNA was detected in nasal swabs of all NHPs within the first week of infection (a and $\mathbf{b}$ ).

$66{ }^{*}=$ undetectable virus; \# = test was inconclusive (a). Virus was detected in brain endothelial cells,

67 as shown by colocalization of VWF (c) with SARS-N (d). Nuclear labeling with DAPI (e) and merged images (f) are shown. Merged image is also presented in Fig. $\mathbf{4 b}$. Arrows indicate SARS$\mathrm{N}$ positive signal in the endothelium (d). Scale bars $=100 \mu \mathrm{m}$. VL - viral load, PI - post infection, RM - Rhesus macaque, AGM - African green monkey. 


\begin{tabular}{ccccccc}
\hline $\begin{array}{c}\text { Animal } \\
\text { ID }\end{array}$ & $\begin{array}{c}\text { Age } \\
\text { (years) }\end{array}$ & Sex & Species & $\begin{array}{c}\text { Route of } \\
\text { challenge }\end{array}$ & $\begin{array}{c}\text { Virus } \\
\text { exposure }\end{array}$ & $\begin{array}{c}\text { Length of } \\
\text { infection } \\
\text { (days) }\end{array}$ \\
\hline RM1 & 14.01 & Male & M. mulatta & Multi-route & $3.61 \times 10_{6}$ PFU & 27 \\
\hline RM2 & 12.97 & Female & M. mulatta & Multi-route & $3.61 \times 10_{6}$ PFU & 27 \\
\hline AGM1 & 16.3 & Male & C.a. sabaeus & Multi-route & $3.61 \times 10_{6}$ PFU & 26 \\
\hline AGM2 & 16.29 & Female & C.a. sabaeus & Multi-route & $3.61 \times 10_{6}$ PFU & 22 \\
\hline RM3 & 13.06 & Male & M. mulatta & Aerosol & $2 \times 10_{3}$ TCID50* & 28 \\
\hline RM4 & 15.03 & Male & M. mulatta & Aerosol & $2 \times 10_{3}$ TCID50* & 28 \\
\hline AGM3 & 16.33 & Male & C.a. sabaeus & Aerosol & $2 \times 10_{3}$ TCID50* & 24 \\
\hline AGM4 & 16.28 & Female & C.a. sabaeus & Aerosol & $2 \times 10_{3}$ TCID50* & 8 \\
\hline
\end{tabular}

Extended Data Table 1. Study Animals. Four Rhesus macaques and 4 African green monkeys were exposed to SARS-CoV-2 strain 2019-nCoV/USA-WA1/2020 by multiple mucosal routes or aerosolized virus in an aerosol chamber. All animals survived to study endpoint at 24-28 days post infection, with the exception of AGM4 and AGM2 who reached humane endpoints at 8- and 22-days post infection, respectively. *Approximate inhaled dose. PFU = plaque-forming unit; TCID $_{50}=50 \%$ tissue culture infectious dose; $\boldsymbol{M}$. mulatta = Macaca mulatta (Rhesus macaque, RM); C.a. sabaeus = Chlorocebus aethiops sabaeus (African green monkey, AGM) 


\begin{tabular}{|c|c|c|c|}
\hline ID & $\begin{array}{c}\text { Age } \\
\text { (years) }\end{array}$ & Sex & CNS pathology and summary of findings \\
\hline RM1 & 14.01 & Male & $\begin{array}{l}\text { Multiple acute microhemorrhages (++++) were observed in cerebellum, BG, and } \\
\text { brainstem. Marked neuronal and surrounding cell injury was seen within } \\
\text { cerebellum and brainstem. Cleaved caspase } 3 \text { positivity was mostly limited to } \\
\text { Purkinje cells and immediate neighbors (+++) in cerebellum. BG had a single } \\
\text { region of cell with sporadic caspase } 3 \text { positivity within the area. Limited vascular } \\
\text { caspase } 3 \text { positivity was also observed in the BG }(+) \text {. No caspase } 3 \text { positivity } \\
\text { was observed in brainstem despite large regions with abnormal neuronal } \\
\text { morphology. Limited SARS-N positivity was found in endothelium of cerebellum, } \\
\text { brainstem, and BG (+). }\end{array}$ \\
\hline RM2 & 12.97 & Female & $\begin{array}{l}\text { Acute microhemorrhages were seen in cerebellum and BG (+). Marked neuronal } \\
\text { injury was present in cerebellum and brainstem. Cleaved caspase } 3 \text { positivity } \\
\text { was observed in Purkinje cells and immediate neighbors in cerebellum and } \\
\text { parenchymal cells in brainstem }(+++) \text {. Rare caspase } 3 \text { positivity was observed } \\
\text { in cerebellar endothelium }(1 / 2+) \text {, with much greater EC positivity seen in } \\
\text { brainstem }(+++) \text {. Caspase } 3 \text { was also observed in BG endothelium, but to a } \\
\text { lesser degree }(+) \text {. BG also showed limited caspase } 3 \text { positivity of parenchymal } \\
\text { cells with apparent nuclear dissolution and surface blebs }(+) \text {. Parietal lobe } \\
\text { showed rare cleaved caspase } 3 \text { positivity in ECs and parenchymal cells }(1 / 2+) \text {. } \\
\text { This was localized to blood vessels within associated areas of tissue damage } \\
\text { that contained cells at different stages of nuclear dissolution with apparent } \\
\text { blebbing. The temporal lobe had several foci with high cleaved caspase } 3 \\
\text { positivity }(+++) \text {. Cleaved caspase } 3 \text { was also seen with moderate frequency in } \\
\text { temporal lobe ECs (++). Limited SARS-N positivity in endothelium of cerebellum } \\
\text { and brainstem }(+) \text {, with infrequent positivity observed in BG }(1 / 4+) \text {. }\end{array}$ \\
\hline AGM1 & 16.3 & Male & $\begin{array}{l}\text { Several acute microhemorrhages }(+++) \text { were seen in cerebellum, BG, and } \\
\text { brainstem. Marked neuronal caspase } 3 \text { positivity was seen in Purkinje cells and } \\
\text { immediate neighboring cells }(+++) \text { within the cerebellum, whereas EC- } \\
\text { associated positivity was rare }(1 / 2+) \text {. Considerable caspase } 3 \text { positivity was } \\
\text { present in parenchymal and ECs of brainstem }(++++) \text { and parietal lobe } \\
\text { [parenchymal }(+++) \text {; ECs }(+)] \text {. Rare SARS-N positivity was observed in the } \\
\text { endothelium of cerebellum, brainstem, BG, and temporal lobe }(1 / 2+) \text {, which was } \\
\text { infrequent and dim in the occipital lobe }(1 / 4+) \text {. }\end{array}$ \\
\hline AGM2 & 16.29 & Female & $\begin{array}{l}\text { A considerable number of acute microhemorrhages }(++++) \text { were observed in } \\
\text { cerebellum, BG, and brainstem. While marked neuronal injury was observed in } \\
\text { cerebellum, cleaved caspase } 3 \text { positivity in Purkinje cells and immediate } \\
\text { neighbors was moderate }(++) \text {. Brainstem had foci of caspase } 3 \text { positivity }(++) \text {, } \\
\text { whereas the parietal lobe contained regions of apparent cell injury/death without } \\
\text { cleaved caspase } 3 \text { positivity. Rare SARS-N positivity was noted in endothelium } \\
\text { of cerebellum, brainstem, BG, and parietal lobe }(1 / 2+) \text {. }\end{array}$ \\
\hline RM3 & 13.06 & Male & $\begin{array}{l}\text { An acute microhemorrhage }(1 / 4+) \text { was seen in the BG but not in cerebellum or } \\
\text { brainstem, in contrast to the majority of our study animals. Moderate neuronal } \\
\text { injury with vacuoles in WM were seen in cerebellum. Rare cleaved caspase } 3 \\
\text { positivity was seen in Purkinje cells and immediate neighbors }(1 / 2+) \text {. Very rare } \\
\text { ferric iron positivity was seen in cerebellar WM }(1 / 4+) \text {. Limited pyknotic neurons } \\
\text { were observed in brainstem, however, infrequent caspase } 3 \text { positivity was } \\
\text { restricted to the endothelium }(1 / 2+) \text {. Rare cleaved caspase } 3 \text { positivity was also } \\
\text { observed in parietal lobe, despite apparent areas of cell injury/death. Rare } \\
\text { SARS-N positivity was detected in endothelium of brainstem, BG, and parietal } \\
\text { and temporal lobes }(1 / 2+) \text {. }\end{array}$ \\
\hline
\end{tabular}




\begin{tabular}{|c|c|c|c|}
\hline ID & $\begin{array}{c}\text { Age } \\
\text { (years) }\end{array}$ & Sex & CNS pathology and summary of findings \\
\hline RM4 & 15.03 & Male & $\begin{array}{l}\text { A moderate number of acute microhemorrhages }(++) \text { were seen in cerebellum, } \\
\text { BG, and brainstem. Marked neuronal injury was observed in cerebellum with WM } \\
\text { vacuolation. Active caspase } 3 \text { positivity was not detected. In brainstem, foci of } \\
\text { cell injury/apoptosis were seen with active caspase } 3 \text { positivity in parenchymal } \\
\text { cells and endothelium }(++) \text {. Rare SARS-N positivity was found in endothelium of } \\
\text { cerebellum and temporal lobe }(1 / 2+) \text {, with infrequent positivity in parietal and } \\
\text { occipital lobes }(1 / 4+) \text {. }\end{array}$ \\
\hline AGM3 & 16.33 & Male & $\begin{array}{l}\text { Several acute microhemorrhages }(+++) \text { were seen in cerebellum and brainstem. } \\
\text { Cerebellum showed marked neuronal injury with moderate cleaved caspase } 3 \\
\text { positivity in Purkinje cells and immediate neighbors }(++) \text {. Rare caspase } 3 \\
\text { positivity was seen in cerebellar and BG endothelial cells }(1 / 4+) \text {. Focal regions } \\
\text { of parenchymal cell injury were present in brainstem with active caspase } 3 \\
\text { positivity in parenchymal cells }(1 / 2+) \text { and ECs }(+) \text {. A single region within the } \\
\text { parietal lobe had considerable cleaved caspase } 3 \text { positivity in the parenchyma } \\
(++++ \text { for this area only). Limited SARS-N positivity was seen in endothelium of } \\
\text { cerebellum, brainstem, and BG }(+) \text {, which was infrequent in temporal, parietal, } \\
\text { and occipital lobe endothelium }(1 / 4+) \text {. }\end{array}$ \\
\hline AGM4 & 16.28 & Female & $\begin{array}{l}\text { Extensive acute microhemorrhages }(++++) \text { were seen in cerebellum, BG, and } \\
\text { brainstem. Marked neuronal and neighboring cell injury were also seen in } \\
\text { cerebellum, BG, and brainstem but without cleaved caspase } 3 \text { positivity. } \\
\text { Likewise, cleaved caspase } 3 \text { was not seen in parietal lobe, despite obvious } \\
\text { cell/tissue injury and/or death. Rare SARS-N positivity was detected in } \\
\text { endothelium of cerebellum, brainstem, and BG }(1 / 2+) \text {, which was infrequent and } \\
\text { dim in the temporal lobe }(1 / 4+) \text {. }\end{array}$ \\
\hline RM5 & 14.73 & Female & $\begin{array}{l}\text { Rare vacuoles in parietal lobe and microhemorrhages in brainstem }(1 / 4+) \text { were } \\
\text { noted. Archived BG was not available for this animal. All investigated brain } \\
\text { regions were negative for cleaved caspase } 3 \text {. }\end{array}$ \\
\hline RM6 & 10.76 & Female & $\begin{array}{l}\text { Rare microhemorrhages }(1 / 4+) \text { were noted within the brainstem and cerebellum. } \\
\text { Archived BG was not available for this animal. Rare cleaved caspase } 3 \text { positivity } \\
\text { was observed in brainstem parenchymal cells and ECs }(1 / 4+) \text { within a single } \\
\text { infarcted area. An area of blood vessel associated injury was seen in the } \\
\text { temporal lobe without caspase } 3 \text { positivity. All other investigated brain regions } \\
\text { were negative for cleaved caspase } 3 \text {. }\end{array}$ \\
\hline
\end{tabular}

Extended Data Table 2. CNS Pathology and Summary of Findings. A within laboratory scoring scale, ranging from limited $(+)$, mild $(++)$, moderate $(+++)$, and severe $(++++)$ indicates the degree of positivity of specific antigens investigated or severity of observed pathology. BG - basal ganglia, WM - white matter, ECs endothelial cells, $\mathbf{R M}=$ Rhesus macaque, $\mathbf{A G M}=$ African green monkey 


\section{Figures}
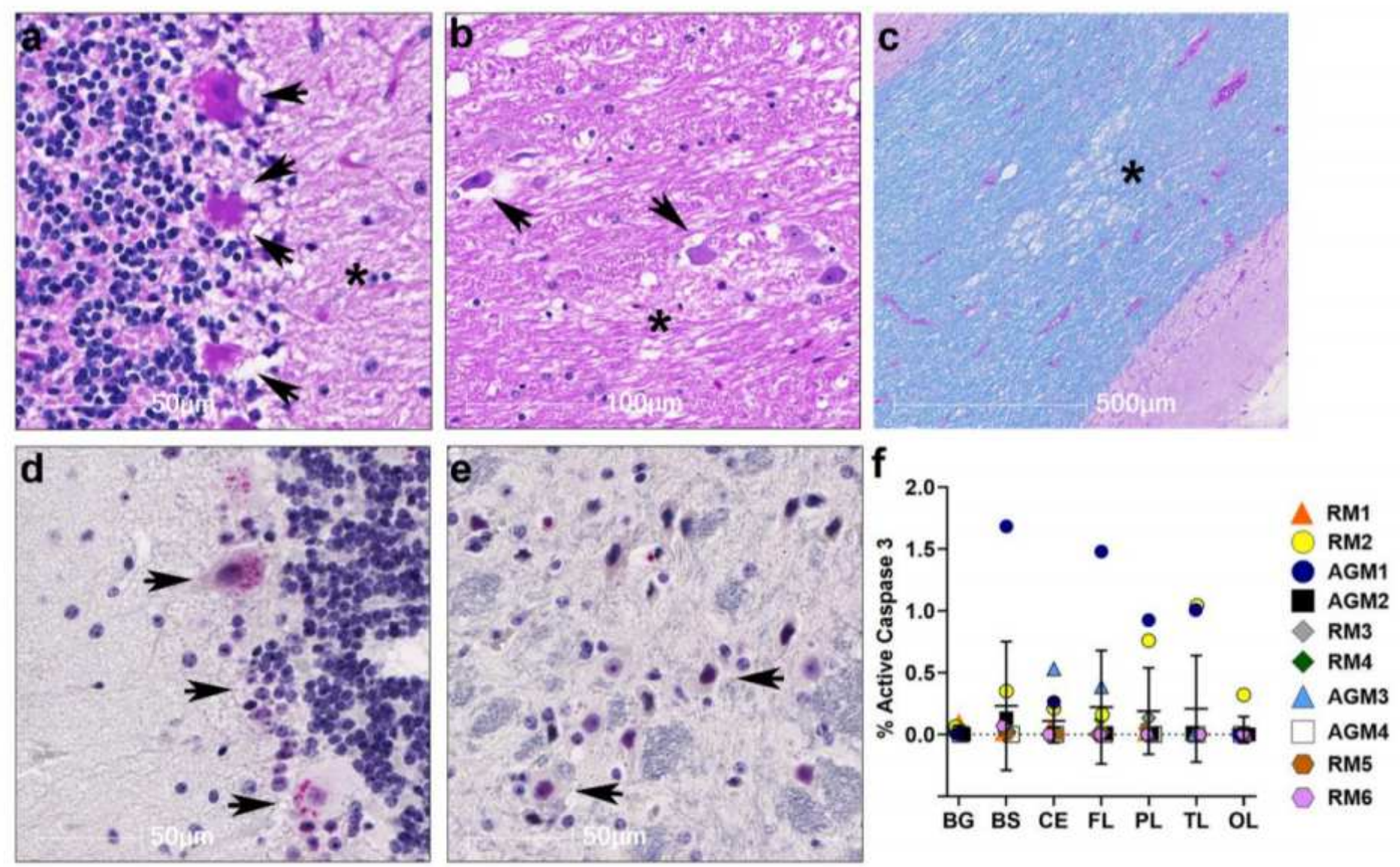

Figure 1

. CNS cell death in SARS-CoV-2 infection. H\&E revealed death-associated neurons in cerebellum (a) and brainstem (b). Arrows specify pyknotic and karyolitic cells ( $a$ and $b$ ) and Purkinje cells with cellular blebs (a). Asterisks denote areas of tissue necrosis/vacuolation ( $a$ and $b$ ) and myelin vacuolation on Luxol Fast Blue stained cerebellum (c). Cleaved caspase 3 positive cells were found in cerebellum (d) and brainstem (e). Summary of cleaved caspase 3 frequency is organized by brain region (f). Data are expressed as mean \pm SD. BG - basal ganglia, BS - brainstem, CE - cerebellum, FL - frontal lobe, PL - parietal lobe, TL temporal lobe, OL - occipital lobe, RM - Rhesus macaque, AGM - African green monkey. 

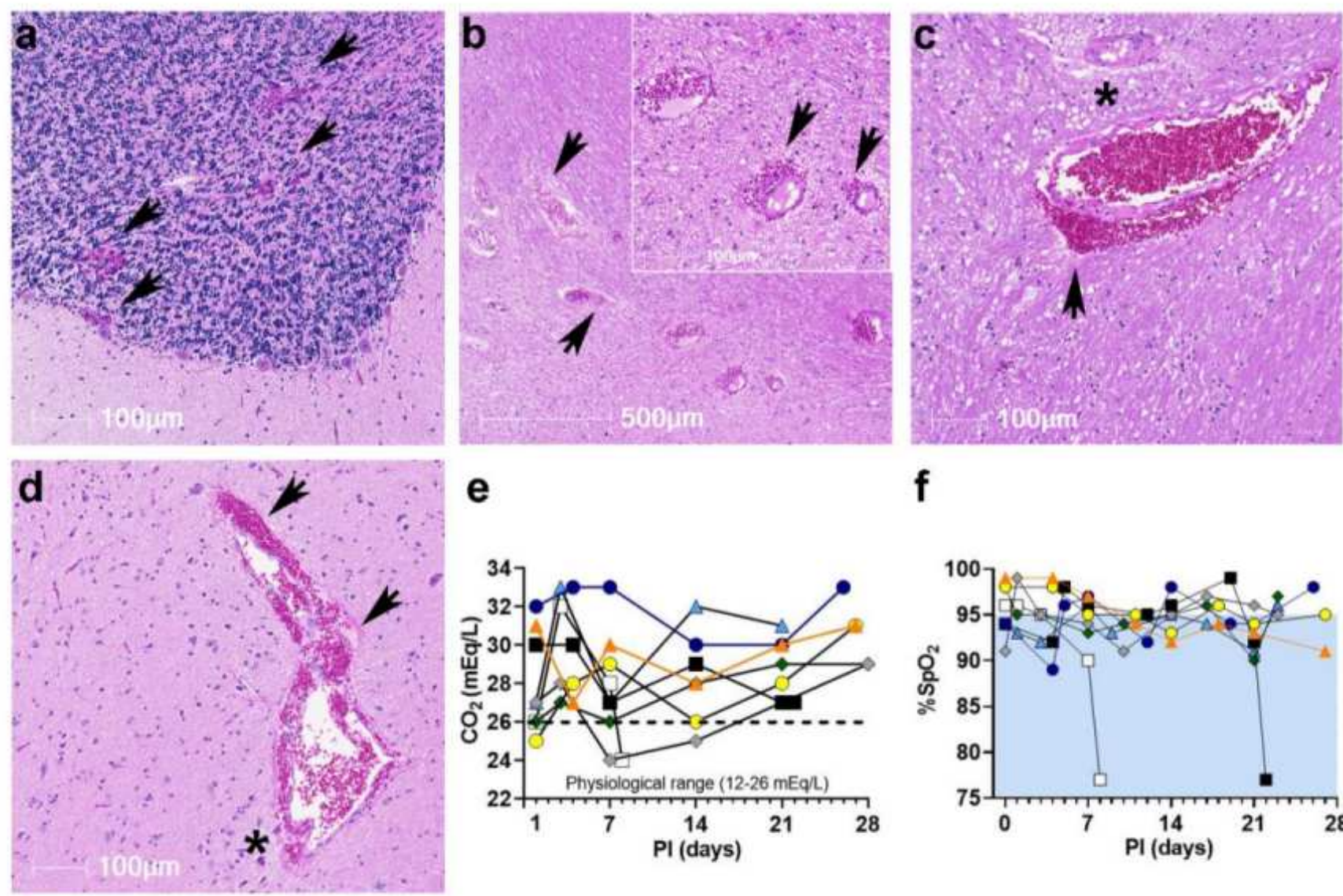

e

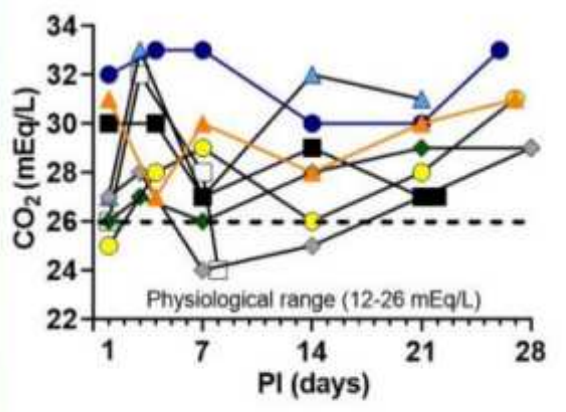

$\mathbf{f}$

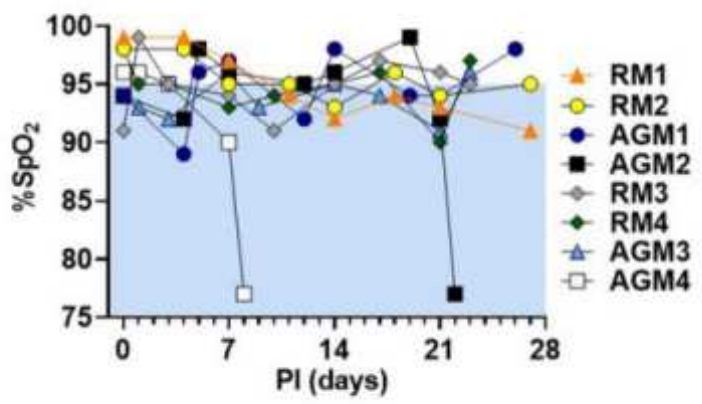

\section{Figure 2}

Multiple microhemorrhages in CNS of SARS-CoV-2 infected NHPs. H\&E of cerebellum (a), brainstem (b and c), and basal ganglia (d) revealed microhemorrhages, indicated by extravasation of erythrocytes into the brain parenchyma (arrows). Asterisks denote tissue injury around damaged blood vessels. Elevated blood CO2 (> $26 \mathrm{mEq} / \mathrm{L}$ ) was detected throughout the study period for most animals. Dotted line indicates the upper limit of the physiological range for blood $\mathrm{CO} 2$ levels (e). SpO2 remained within the low normal range or under $95 \%$ for the majority of animals throughout the study. Blue shading indicates values $\leq 95 \%$ (f). RM - Rhesus macaque, AGM - African green monkey. 

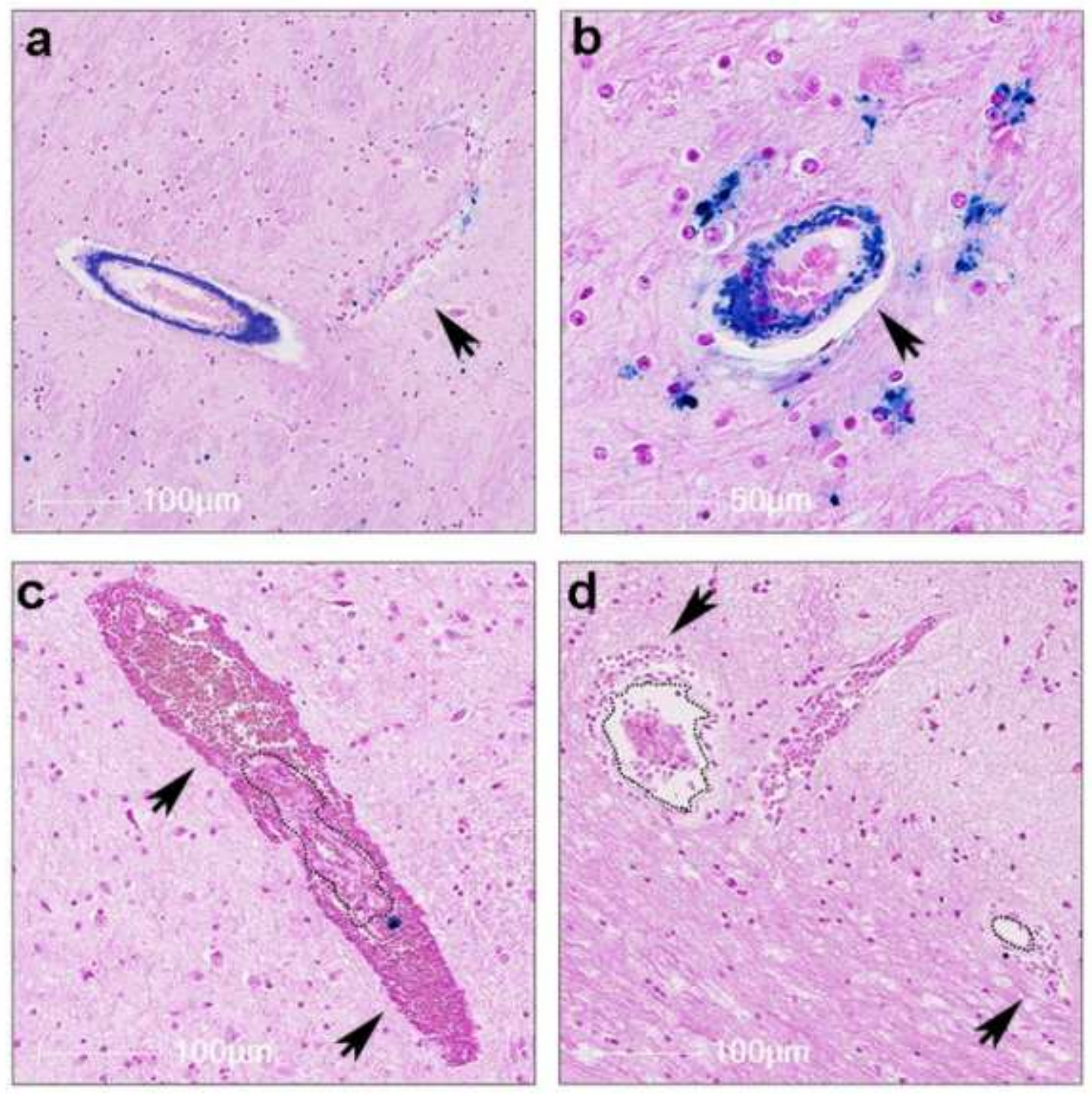

\section{Figure 3}

Pre-existing and acute vascular injury and microbleeds in brain. Gomori Prussian Blue Fe (G Fe) revealed iron deposition within the basal ganglia ( $a$ and $b$ ). Arrow denotes a normal appearing blood vessel in close proximity to an iron positive vessel (a). Iron deposition was predominantly seen in blood vessels with rare parenchymal involvement (b). Acute microhemorrhages were also identified on $\mathrm{G}$ Fe stained basal ganglia, indicated by the absence of G Fe positivity (arrows, $c$ and d). Dotted lines outline the blood vessel within the hemorrhagic lesion (c and d). 

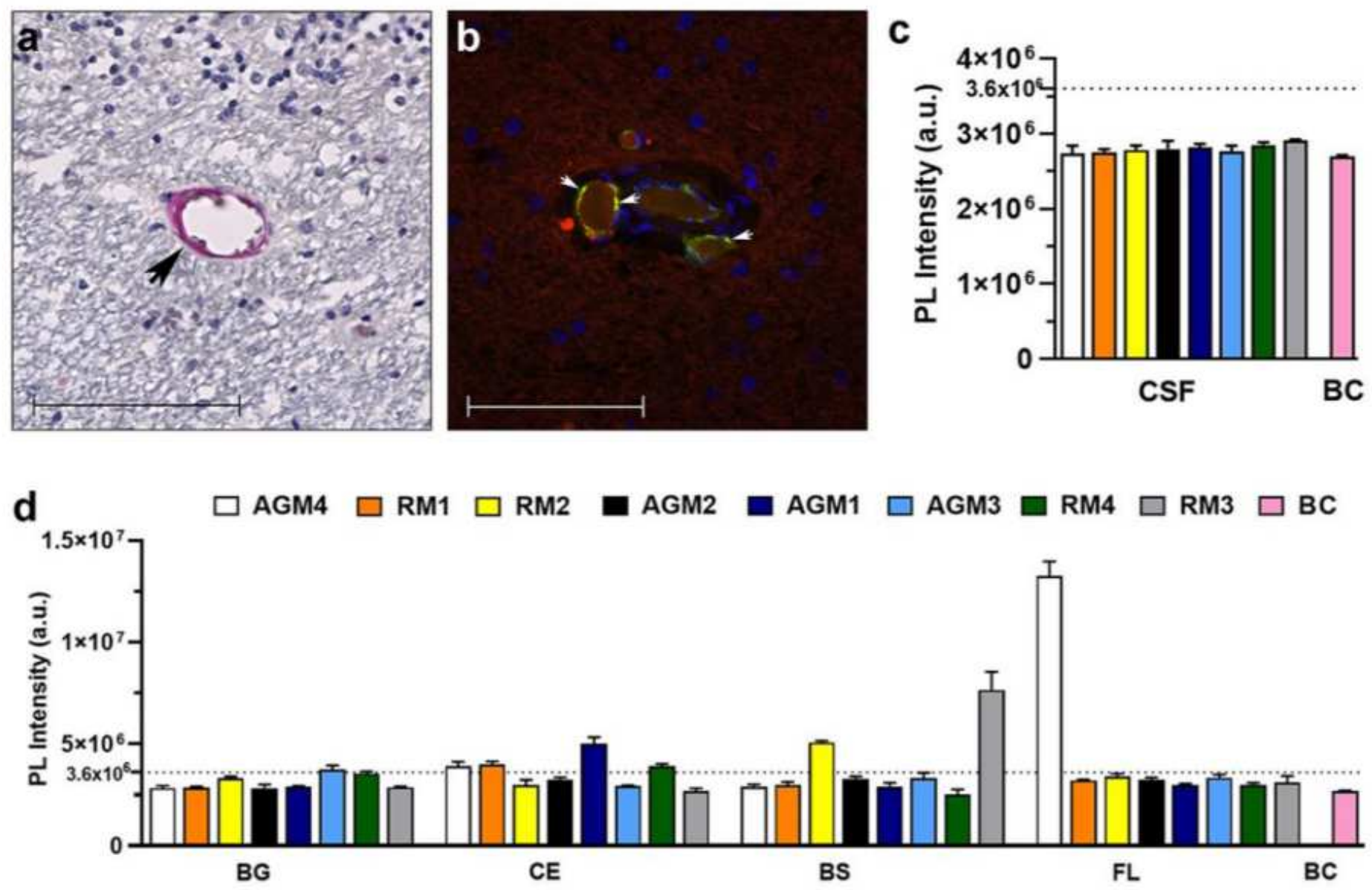

Figure 4

SARS-CoV-2 in the brain. IHC shows SARS-CoV-2 nucleocapsid (SARS-N) in cerebellar blood vessels (a). Endothelial cell infection was confirmed by colocalization of SARS- $\mathrm{N}$ (red) and von Willebrand Factor (vWF) (green) (arrows, b). Scale bars $=100$ Im. SARS-CoV- 2 RNA was undetectable in CSF (c) but was detected in different brain regions investigated (d). Values above 3.6×106 PL intensity (dotted line) were considered positive. a.u. - arbitrary unit; CSF - cerebrospinal fluid, BC - blank control, BG - basal ganglia, CE - cerebellum, BS - brainstem, FL - frontal lobe, RM - Rhesus macaque, AGM - African green monkey. 